\title{
General Model for Light Curves of Chromospherically Active Binary Stars
}

\author{
Jetsu, L.
}

2017-03-31

Jetsu , L , Henry , G W \& Lehtinen , J 2017 , ' General Model for Light Curves of

Chromospherically Active Binary Stars ' , Astrophysical Journal , vol. 838 , no. 2 , pp. 122 . https://doi.org/10.3847/15

http://hdl.handle.net/10138/178886

https://doi.org/10.3847/1538-4357/aa65cb

unspecified

publishedVersion

Downloaded from Helda, University of Helsinki institutional repository.

This is an electronic reprint of the original article.

This reprint may differ from the original in pagination and typographic detail.

Please cite the original version. 


\title{
General Model for Light Curves of Chromospherically Active Binary Stars*
}

\author{
L. Jetsu ${ }^{1}$, G. W. Henry ${ }^{2}$, and J. Lehtinen ${ }^{3}$ \\ ${ }^{1}$ Department of Physics, FI-00014 University of Helsinki, Finland; lauri.jetsu@ @elsinki.fi \\ ${ }^{2}$ Center of Excellence in Information Systems, Tennessee State University, Nashville, TN 37209, USA \\ ${ }_{3}^{3}$ Max-Planck-Institut für Sonnensystemforschung, D-37077 Göttingen, Germany \\ Received 2016 December 6; revised 2017 February 20; accepted 2017 March 5; published 2017 March 31
}

\begin{abstract}
The starspots on the surface of many chromospherically active binary stars concentrate on long-lived active longitudes separated by $180^{\circ}$. Shifts in activity between these two longitudes, the "flip-flop" events, have been observed in single stars like FK Comae and binary stars like $\sigma$ Geminorum. Recently, interferometry has revealed that ellipticity may at least partly explain the flip-flop events in $\sigma$ Geminorum. This idea was supported by the double-peaked shape of the long-term mean light curve of this star. Here we show that the long-term mean light curves of 14 chromospherically active binaries follow a general model that explains the connection between orbital motion, changes in starspot distribution, ellipticity, and flip-flop events. Surface differential rotation is probably weak in these stars, because the interference of two constant period waves may explain the observed light curve changes. These two constant periods are the active longitude period $\left(P_{\text {act }}\right)$ and the orbital period $\left(P_{\text {orb }}\right)$. We also show how to apply the same model to single stars, where only the value of $P_{\text {act }}$ is known. Finally, we present a tentative interference hypothesis about the origin of magnetic fields in all spectral types of stars.
\end{abstract}

Key words: binaries: general - methods: data analysis - stars: activity - techniques: photometric

Supporting material: data behind figures

\section{Introduction}

An ancient Egyptian calendar of lucky and unlucky days, the Cairo Calendar, is the oldest preserved historical document of the discovery of a variable star, Algol (Porceddu et al. 2008; Jetsu et al. 2013; Jetsu \& Porceddu 2015). Today, the General Catalogue of Variable Stars contains nearly 50,000 stars. There are numerous classes of variable stars, and the classification criteria are constantly updated (Samus et al. 1997). Different classes have their own typical light curves (Drake et al. 2014). One class of variable stars is called the Chromospherically Active Binary Stars (hereafter CABS). The third CABS catalogue lists 409 such binaries (Eker et al. 2008).

In his review of starspots, Strassmeier (2009) wrote that the "starspot hypothesis" was first presented by the French astronomer Ismael Boulliau (1605-1694) to explain the variability of Mira. This star, also known as $o$ Ceti, was the first variable star discovered by "modern" astronomers in 1596 (David Fabricius, 1564-1617). Boulliau's hypothesis was unfortunately not true, because pulsations cause the variability of Mira. According to Strassmeier (2009), the first observations of starspots were made by Kron (1947) in the light curves of four eclipsing binaries. The presence of this phenomenon in the light curves of active stars was firmly established later by Hoffmeister (1965), Chugainov (1966), Catalano \& Rodonò (1967), and Hall (1972).

Unlike the longitudinally uniform distribution of spots on the Sun, the starspots in active single (e.g., Jetsu et al. 1993, FK Com) or binary (e.g., Jetsu 1996, $\sigma$ Gem) stars concentrate at long-lived active longitudes and seem to undergo shifts of about $180^{\circ}$ in longitude. The presence of this "flip-flop" phenomenon in the CABS $\sigma$ Gem was questioned by Roettenbacher et al. (2015). Their interferometric observations revealed that ellipticity of $\sigma$ Gem may explain the stability of

\footnotetext{
* The CPS results are available electronically at the Vizier database.
}

the two minima in the long-term mean light curve (hereafter MLC). Recently, Siltala et al. (2016) reported that another CABS, BM CVn, has a sinusoidal MLC with an amplitude of $0 \mathrm{~m} .042$. Ellipticity fails to explain the MLC shape of BM CVn. Here we study the light curves of fourteen CABS. Our sample includes the light curves of the two previously mentioned CABS, $\sigma$ Gem and BM CVn.

We use the following abbreviations:

CABS $=$ Chromospherically active binary star

MLC $=$ Mean light curve

$\mathrm{CPS}=$ Continuous period search

$\mathrm{A}=$ More active CABS component

$\mathrm{B}=$ Other CABS component

$\mathrm{Aa}=\mathrm{A}$ in front of $\mathrm{B}$ epoch at $\phi_{\text {orb }}=0.75$

$\mathrm{Ac}=\mathrm{B}$ in front of $\mathrm{A}$ epoch at $\phi_{\mathrm{orb}}=0.25$

$\mathrm{Ab}=$ Mid epoch between Aa and Ac at $\phi_{\text {orb }}=0.00$

$\mathrm{Ad}=$ Mid epoch between Ac and Aa at $\phi_{\text {orb }}=0.50$

$\mathrm{S} 1=$ Larger stationary starspot on A

$\mathrm{S} 2=$ Smaller stationary starspot on A

$\mathrm{S} 1 \mathrm{f}=\mathrm{S} 1$ visibility maximum at $\mathrm{Aa}$ and No $\mathrm{S} 2$

$\mathrm{S} 1 \mathrm{~b}=\mathrm{S} 1$ visibility maximum at $\mathrm{Ac}$ and No $\mathrm{S} 2$

$\mathrm{S} 12 \mathrm{fb}=\mathrm{S} 1$ visibility maximum and $\mathrm{S} 2$ unseen at $\mathrm{Aa}$

$\mathrm{S} 12 \mathrm{bf}=\mathrm{S} 1$ unseen and $\mathrm{S} 2$ visibility maximum at $\mathrm{Aa}$

S3 = Larger nonstationary starspot on A

$\mathrm{S} 4=$ Smaller nonstationary starspot on A.

The meaning of the last 12 abbreviations is explained in greater detail in Section 6. We emphasize that the previously listed $\mathrm{Aa}, \mathrm{Ab}, \mathrm{Ac}$, and Ad epochs are used to connect the results in Figures 1-27 unambiguously to the sketches of Figures 28 and 29.

\section{Observations}

We have acquired between 23 and 27 years of time series differential $V$ photometric observations of 14 chromospherically 


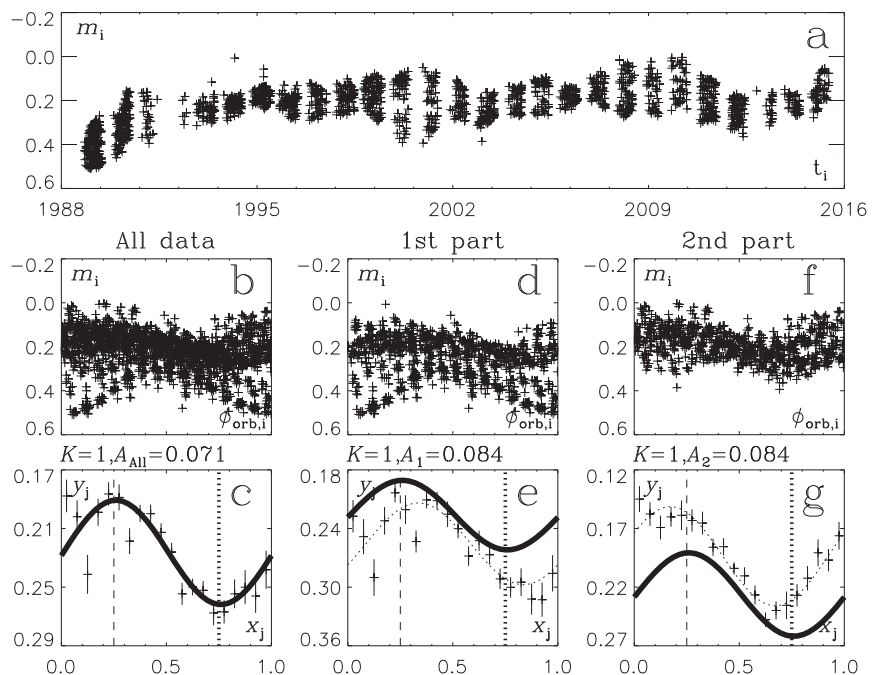

Figure 1. DM UMa. (a) All data: $V$ magnitudes (crosses) vs. time. (b) All data: $V$ magnitudes (crosses) vs. orbital phase. (c) All data: binned magnitudes (crosses with error bars), MLC (thick continuous line), order $(K)$, and amplitude $\left(A_{\text {All }}\right)$. Epoch Aa (thick dotted vertical line) and epoch Ac (thin dashed vertical line). (d) First part of data: $V$ magnitudes (crosses) vs. orbital phase. (e) First part of data: binned magnitudes (crosses with error bars), MLC (thin dashed line), order $(K)$, amplitude $\left(A_{1}\right)$, and MLC of all data (thick line from "a"). Epoch Aa (thick dotted vertical line) and epoch Ac (thin dashed vertical line). (f and g) Second part of data: otherwise as in panels $d$ and e. (The data used to create this figure are available.)

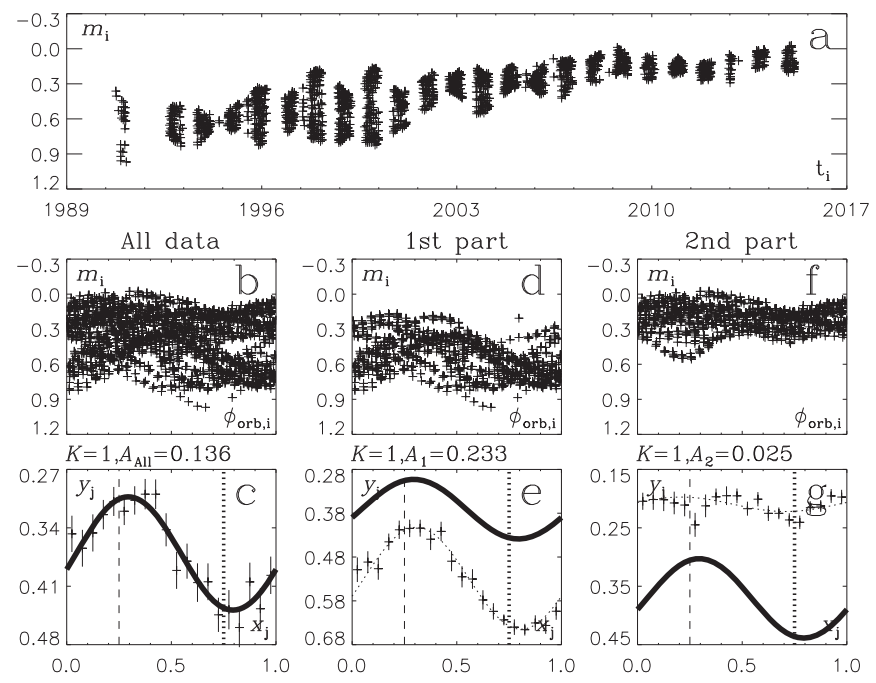

Figure 2. XX Tri. Notice the dips in $(\mathrm{g})$ at $\phi_{\text {orb }} \approx 0.25$ and 0.75 ; otherwise as in Figure 1.

(The data used to create this figure are available.)

active binaries with Tennessee State University's T3 $0.4 \mathrm{~m}$ automatic photometric telescope located at Fairborn Observatory in southern Arizona. The precision of the individual photometric measurements averages around $0 \mathrm{~m} .005$ on good photometric nights (see Henry et al. 1995b, Table 3). A summary of the photometric observations is given in Table 1.

\section{MLC Analysis}

The $P_{\text {orb }}$ and $t_{0}$ values of each CABS (Eker et al. 2008) are given in Table 1. There are five different types of radial

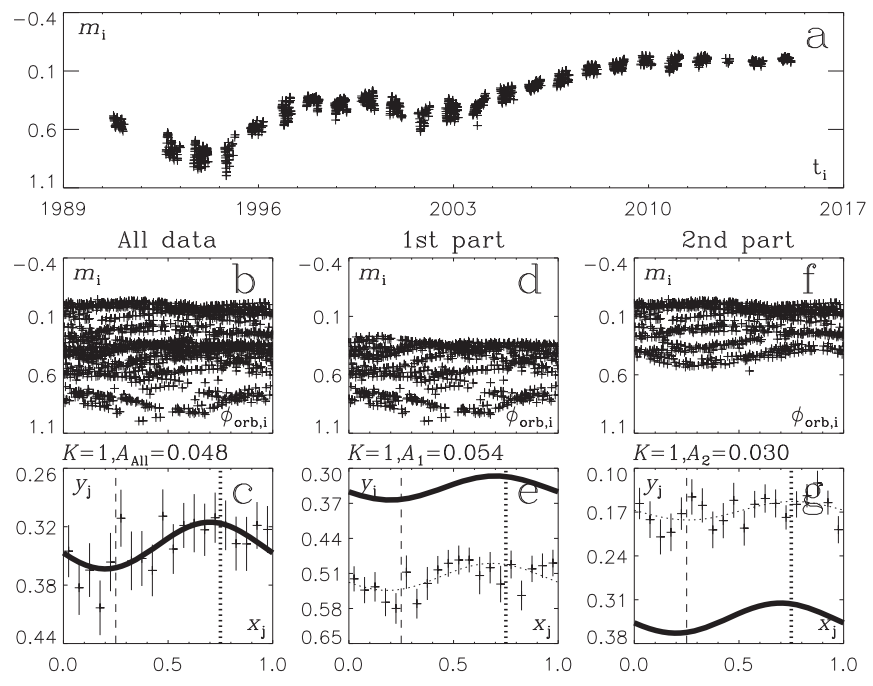

Figure 3. EL Eri, otherwise as in Figure 1.

(The data used to create this figure are available.)

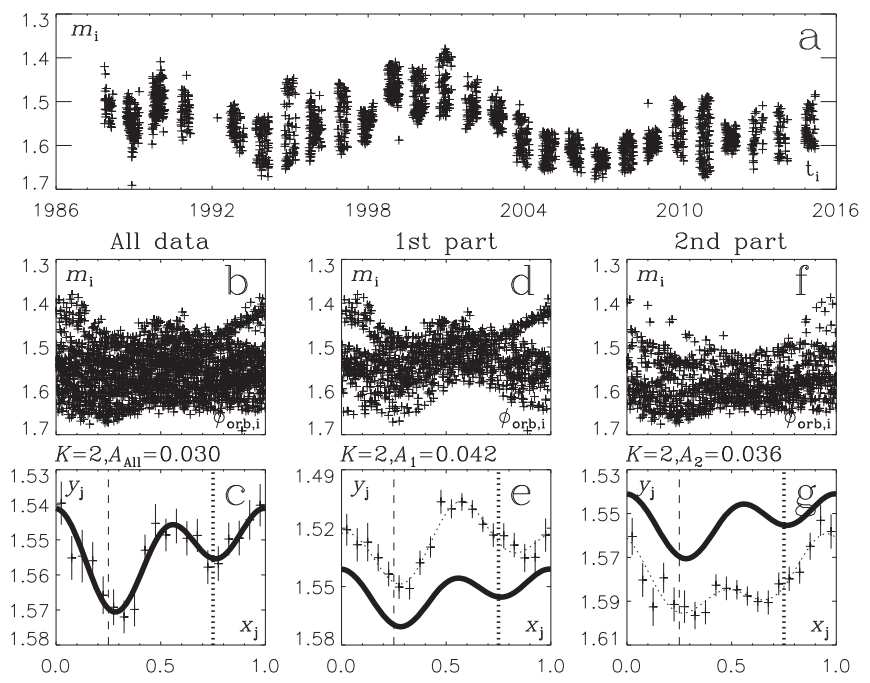

Figure 4. V711 Tau, otherwise as in Figure 1.

(The data used to create this figure are available.)

velocity epochs $t_{0}$ in Table 1 . We use the radial velocity maximum epochs to compute the orbital phases from

$$
\phi_{\text {orb }}=\operatorname{FRAC}\left[\left(t-t_{0}\right) / P_{\text {orb }}\right],
$$

where FRAC removes the integral part of its argument (i.e., the number of full orbital rounds $P_{\text {orb }}$ completed after $t_{0}$ ). Our value for $t_{0}$ is the $\mathrm{Ab}$ epoch given in Table 2 .

The original data are the differential $V$ magnitude observations $m_{i}=m\left(t_{i}\right)$, where $t_{i}$ is the observing time. The time points $t_{i}$ are transformed into phases $\phi_{\text {orb,i }}$ (Equation (1)). The $m_{i}$ observations are binned in phase, using $N=20$ evenly spaced bins, where the limits of the $j$ th bin are $(j-1) / N \leqslant \phi_{\text {orb }, i}<j / N$. A bin must contain at least two $m_{i}$ values. The binned data for the $n_{j}$ values of $m_{i}$ in the $j$ th bin are $x_{j}=(j / N)-1 /$ (2N), $y_{j}=n_{j}^{-1} \sum_{i=1}^{n_{j}} m_{i}, \sigma_{j}=n_{j}^{-1 / 2}\left[n_{j}^{-1} \sum_{i=1}^{n_{j}}\left(m_{i}-y_{j}\right)^{2}\right]^{1 / 2}=$ $n_{j}^{-1}\left[\sum_{i=1}^{n_{j}}\left(m_{i}-y_{j}\right)^{2}\right]^{1 / 2}$. 


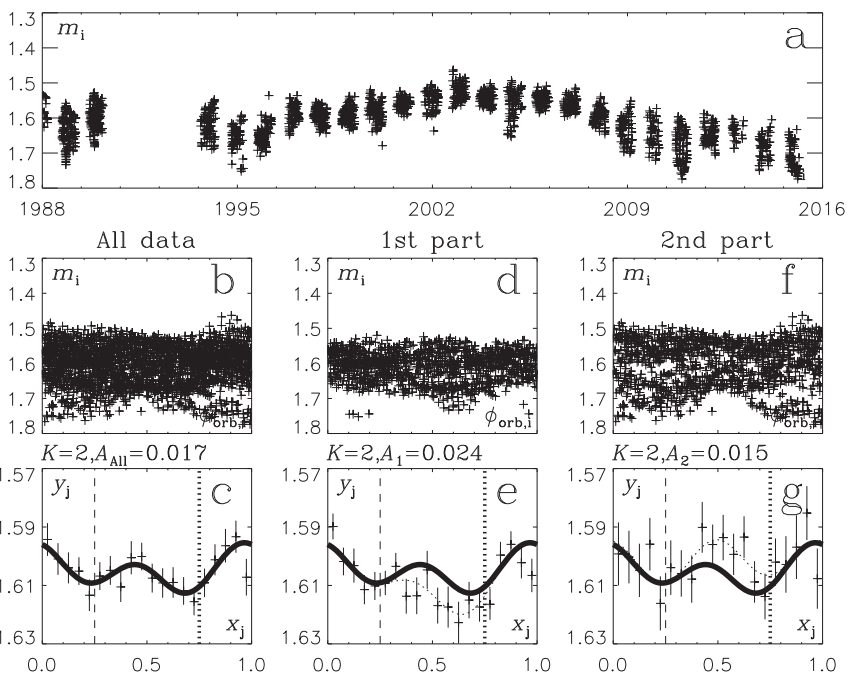

Figure 5. EI Eri. Note that the deeper primary minimum shifts from $\phi_{\text {orb }} \approx 0.65$ to $\approx 0.20$ (eg); otherwise as in Figure 1 .

(The data used to create this figure are available.)

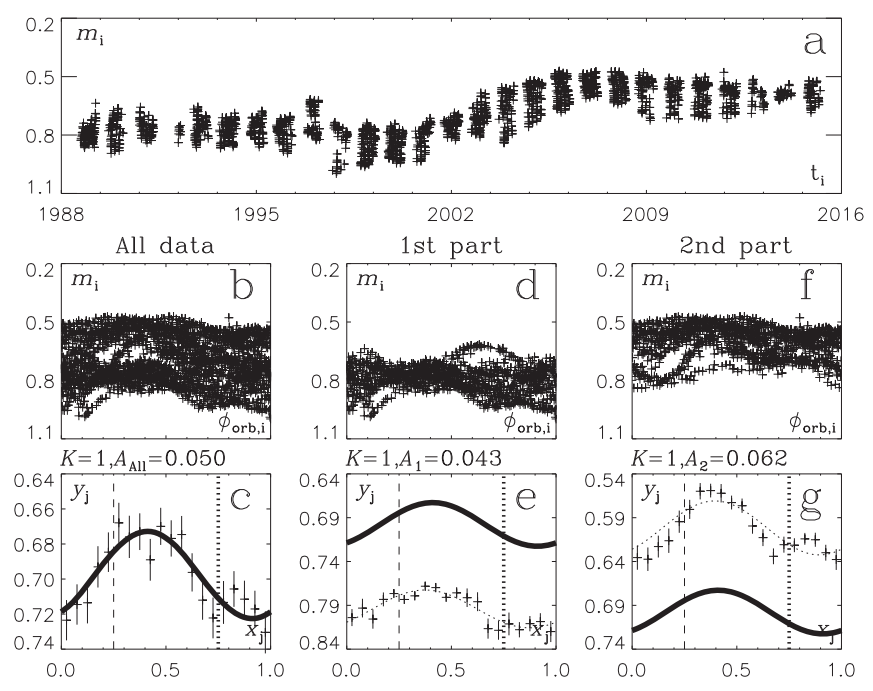

Figure 6. V1149 Ori. Notice the dip in (ceg), at $\phi_{\text {orb }} \approx 0.75$; otherwise as in Figure 1.

(The data used to create this figure are available.)

The model for these data is

$$
g(x, \bar{\beta})=a_{0}+\sum_{k=1}^{K} a_{k} \cos (k x)+b_{k} \sin (k x),
$$

where $\bar{\beta}=\left[a_{0}, a_{1}, \ldots, a_{K}, b_{1}, \ldots, b_{K}\right]$ are the free parameters. Note that the model is simply $g(x, \bar{\beta})=a_{0}$, if $K=0$. The model residuals $\epsilon_{j}=y_{j}-g\left(x_{j}, \bar{\beta}\right)$ give $\chi^{2}(\bar{y}, \bar{\beta})=\sum_{j=1}^{N} w_{j} \epsilon_{j}^{2}$, where $w_{j}=\sigma_{j}^{-2}$.

The main question is, do these data contain a periodic signal? And if so, what is the correct order $K$ for the model of this signal? We solve this problem, as Lehtinen et al. (2011) did, by computing the Bayesian information criterion parameter

$$
R_{\mathrm{BIC}}=2 n \ln \lambda(\bar{y}, \bar{\beta})+(5 K+1) \ln n,
$$

where $\lambda(\bar{y}, \bar{\beta})=\chi^{2}(\bar{y}, \bar{\beta})\left[\sum_{j=1}^{N} w_{j}\right]^{-1}$. This parameter can be used to decide which particular order $K$ gives the best model for the data. The value of $R_{\mathrm{BIC}}$ increases for both too low or too

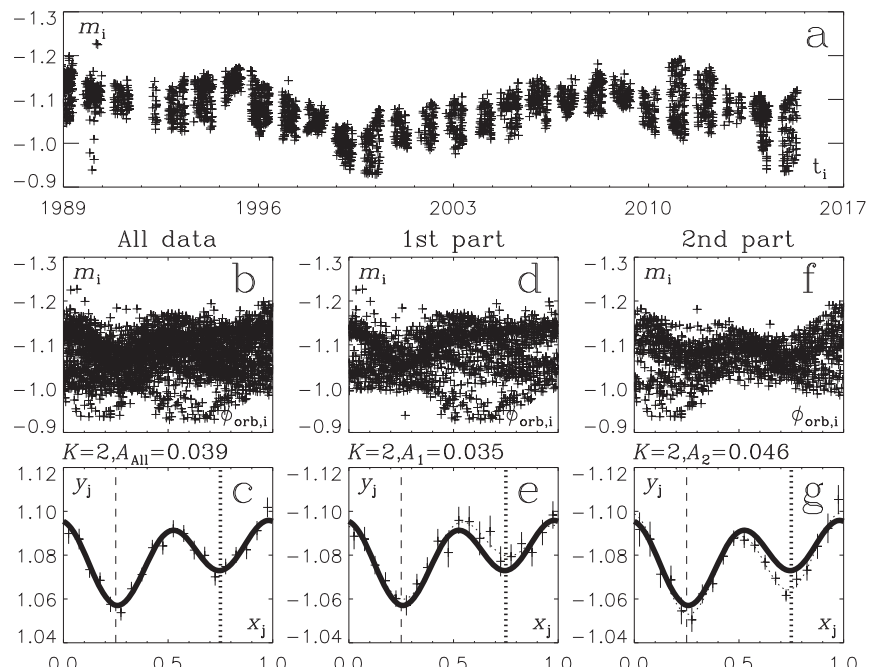

Figure 7. $\sigma$ Gem, otherwise as in Figure 1.

(The data used to create this figure are available.)

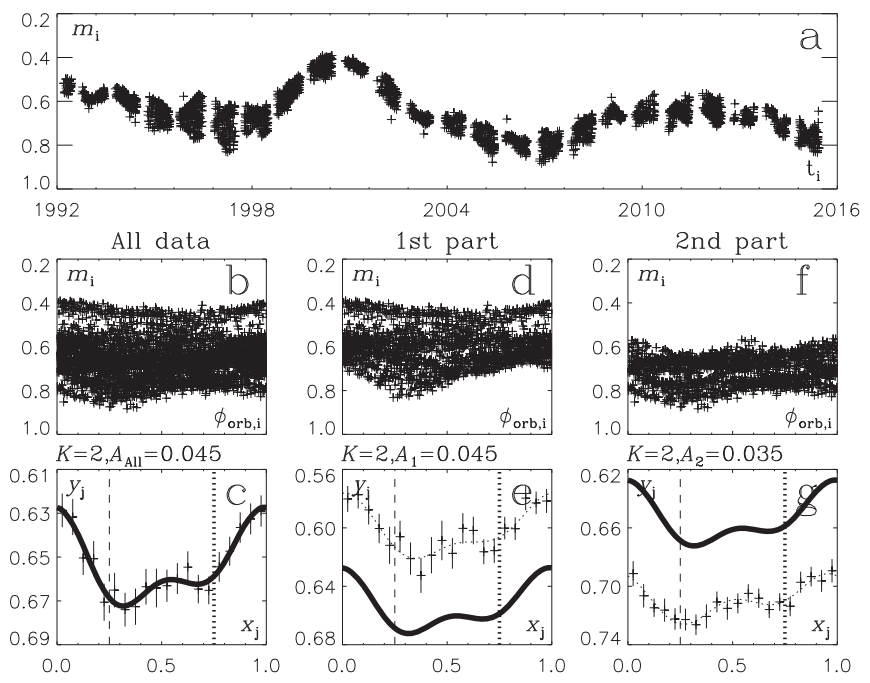

Figure 8. FG UMa; otherwise as in Figure 1.

(The data used to create this figure are available.)

high incorrect values of $K$. The best modeling order $K$ for the data is the value of the order that minimizes $R_{\mathrm{BIC}}$. We test the values $K=0,1$, and 2 for the binned data of each CABS. The best $K$ value for the MLC of all data of each star, and the peak to peak amplitude $A_{\mathrm{All}}$ of this MLC, are given in Table 1. The periodic MLC phenomenon is present in all 14 CABS (i.e., $K \geqslant 1$ ).

We divide the data into two parts in our Figures 1-14. This allows us to check the stability of MLC (i.e., if the starspot distribution changes, then MLC changes). The first and second parts of the data are before and after $t_{1}+\Delta T / 2$, respectively. The order $K$ of the MLC model for the first and second part of the data is fixed to the $K$ value obtained for all data. However, the amplitudes of these MLC models, $A_{1}$ and $A_{2}$, are determined separately from a fit to the binned data of the first and second part of the data. We use the notation $\Delta A$ for the maximum difference between the three amplitudes $A_{\mathrm{All}}, A_{1}$, and $A_{2}$. 


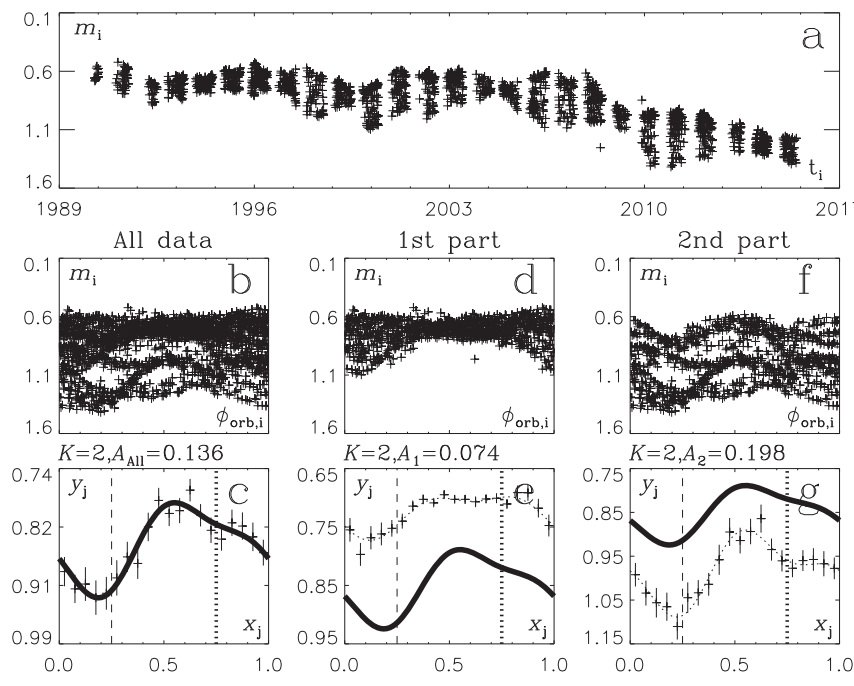

Figure 9. HU Vir; otherwise as in Figure 1.

(The data used to create this figure are available.)

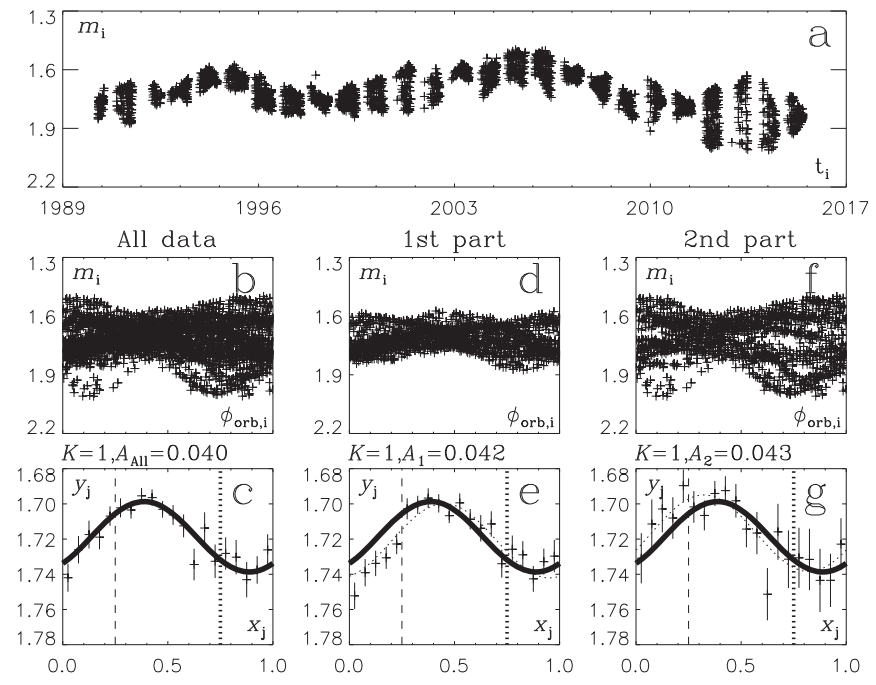

Figure 10. BM CVn; otherwise as in Figure 1.

(The data used to create this figure are available.)

The orbital ephemeris epoch $\left(t_{0}\right)$, the orbital period $\left(P_{0}\right)$, the eccentricity $(e)$, and the spectral type of component A of CABS information in Tables 1 and 2 is from Eker et al. (2008; the third catalogue of CABS). The original references for this information are given in Section 4, where the results for each individual star are discussed separately.

\section{MLC Analysis Results}

Here we describe the MLC of each individual CABS and give the original references for their physical parameters in Tables 1 and 2 .

\subsection{MLC of DM UMa}

The high amplitude, $A_{\mathrm{All}}=0^{\mathrm{m}}$.071, sinusoidal MLC of DM UMa remains nearly unchanged between $0.4<\phi_{\text {orb }}<0.6$ (Figures 1(c), (e) and (g)). The largest MLC changes occur between $\mathrm{Aa} \equiv-0.25<\phi_{\text {orb }}<0.25 \equiv$ Ac. MLC level,

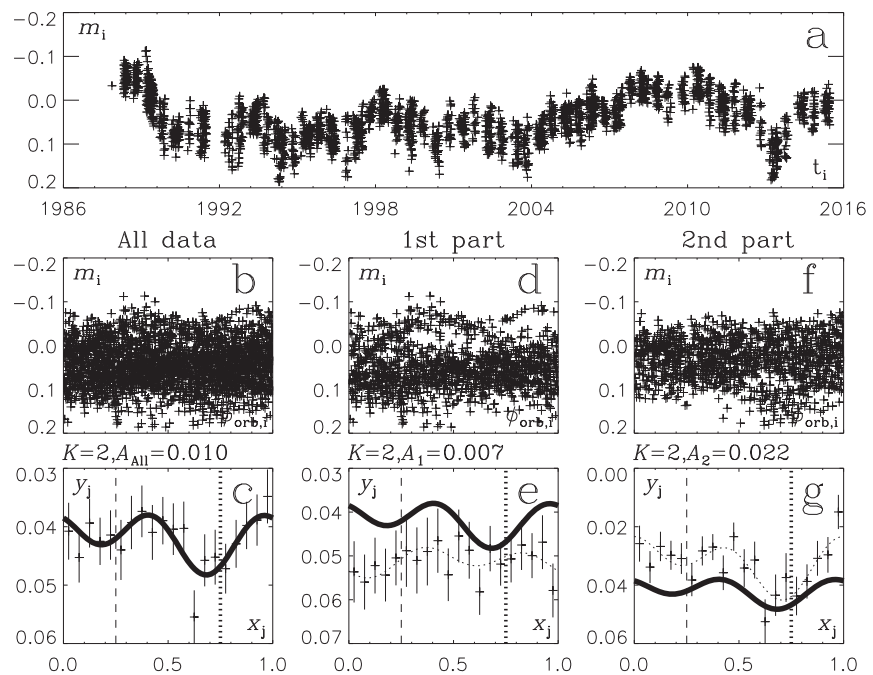

Figure 11. V478 Lyr. Note that the deeper primary minimum shifts from $\phi_{\text {orb }} \approx 0.10$ to $\approx 0.60$ (eg); otherwise as in Figure 1 . (The data used to create this figure are available.)

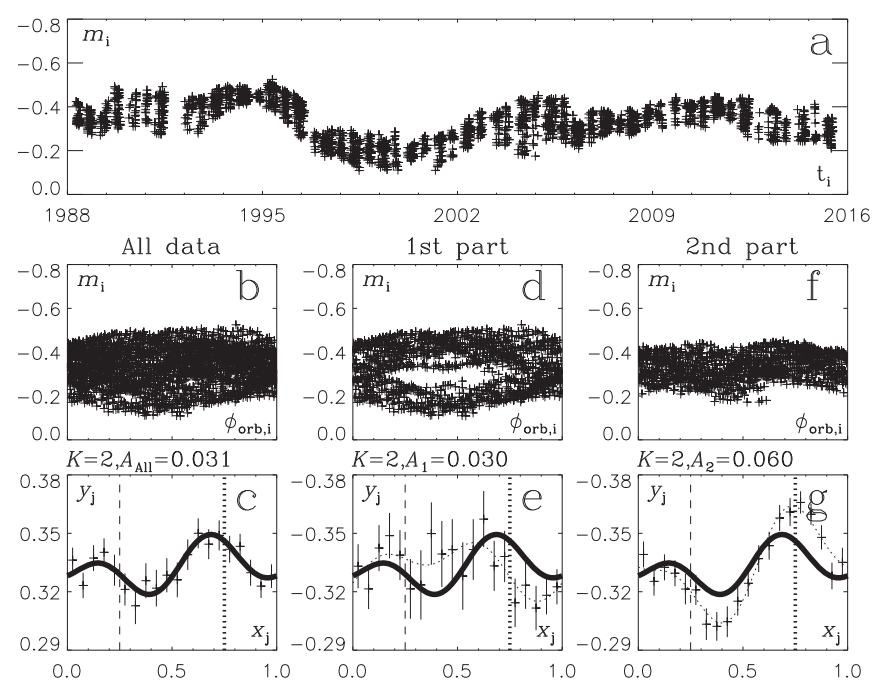

Figure 12. V1762 Cyg; otherwise as in Figure 1. Note that the deeper primary minimum shifts from $\phi_{\text {orb }} \approx 0.90$ to $\approx 0.40$ (eg).

(The data used to create this figure are available.)

shape, and phase are nearly stable. MLC amplitude changes are small $\left(\triangle A=0{ }^{\mathrm{m}} \cdot 013\right)$. MLC minimum coincides with Aa (Crampton et al. 1979; Glebocki \& Stawikowski 1995; Barrado y Navascues et al. 1998; Hatzes $1998, P_{\text {orb }}, t_{0}, e$, Sp-type of component A).

\subsection{MLC of $X X$ Tri}

The sinusoidal MLC of XX Tri has an extremely high amplitude of $A_{\mathrm{All}}=00^{\mathrm{m}}$. 136. This variation increases to $A_{1}=0$ m. 233 during the first part of that data, and then decreases to $A_{2}=0$ m. 025 in the second part. Despite these dramatic changes, the MLC phase remains stable. MLC minimum and maximum phases coincide with $\mathrm{Aa}$ and Ac epochs (Strassmeier \& Olah 1992; Bopp et al. 1993; Strassmeier $1999, P_{\text {orb }}, t_{0}, e$, Sp-type of component A). Note 


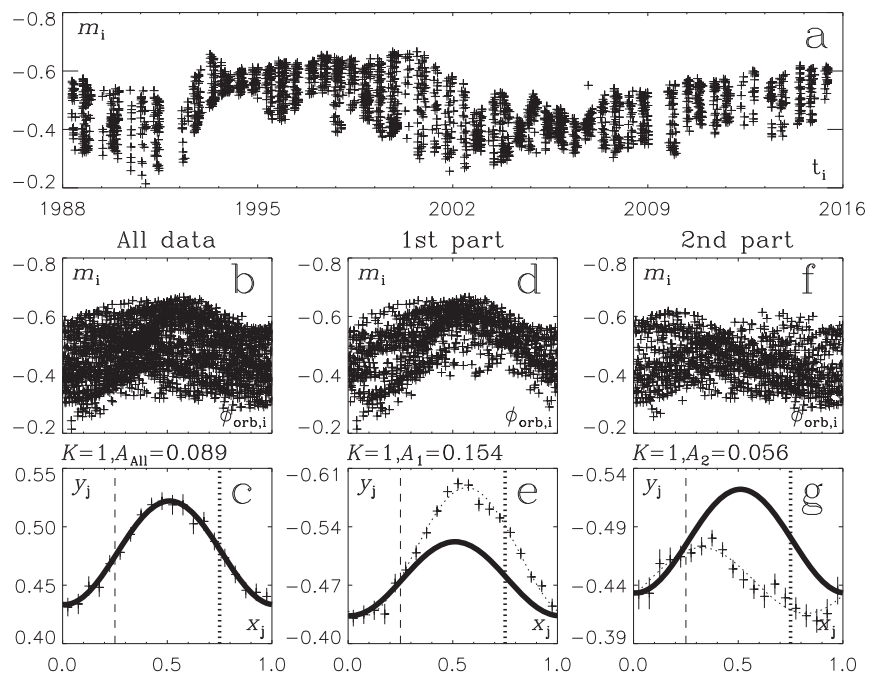

Figure 13. HK Lac; otherwise as in Figure 1.

(The data used to create this figure are available.)

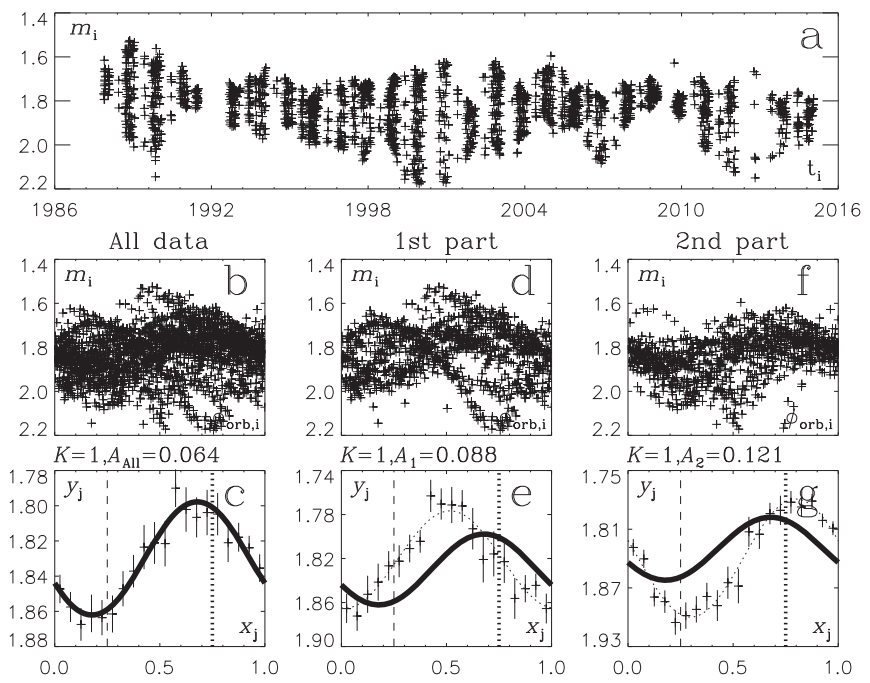

Figure 14. II Peg, otherwise as in Figure 1.

(The data used to create this figure are available.)

the minor MLC dips at $\phi_{\text {orb }}=0.25$ and 0.75 in Figure $2(\mathrm{~g})$, which will be discussed in Section 6.1.4.

\subsection{MLC of EL Eri}

EL Eri has a sinusoidal MLC with an amplitude of $A_{\text {All }}=0$. 048 (Figure 3(c)). MLC shape, amplitude, and phase remain quite stable $\left(\Delta A=0^{\mathrm{m}} .018\right)$, despite the large mean level changes between the first and second part of the data (Figures 3(c)-(g)). The Aa and Ac epochs nearly coincide with the MLC maximum and minimum (Fekel et al. 1986; Balona 1987, $P_{\text {orb }}, t_{0}, e$, Sp-type of component A).

\subsection{MLC of V711 Tau}

The double-peaked MLC of V711 Tau has a low amplitude (Figure $4 ; A_{\text {All }}=0$ m. 030). This amplitude remains quite stable (Figures $4(\mathrm{c}),(\mathrm{e})$, and $(\mathrm{g}) ; \Delta A=0 \mathrm{~m}$.012), regardless of the changes in the mean level (Figures 4(d) and (f)). The MLC primary minimum phase $\phi_{\text {orb }}=0.25$ does not shift, but the

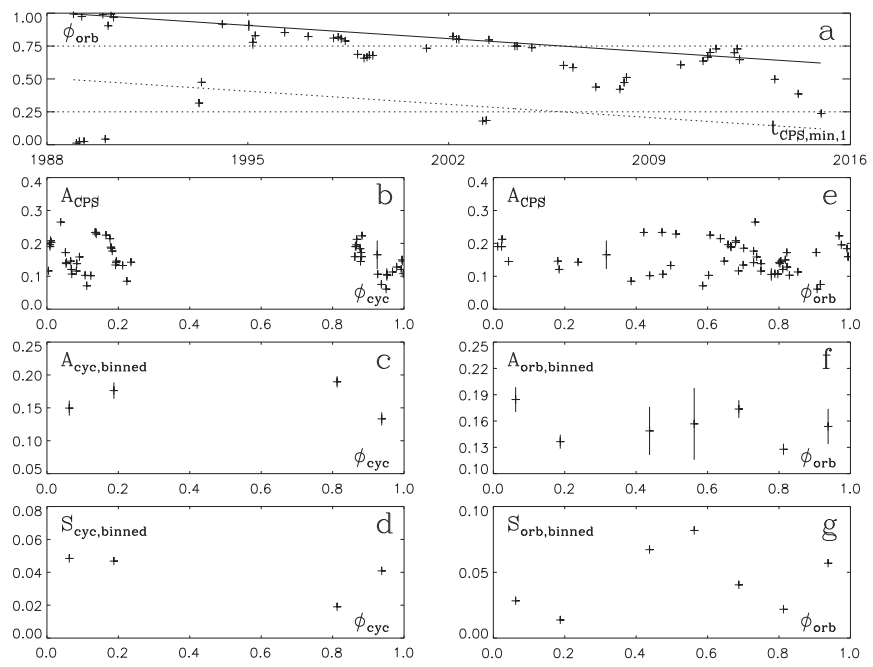

Figure 15. DM UMa. (a) Orbital phases $\phi_{\text {orb }}$ for primary minima $t_{\mathrm{CPS}, \min , 1}$ (note that error bars are shown, but they are smaller than symbol size), orbital phases $\phi_{\text {orb }}=0.25$ and 0.75 (dotted lines), active longitude phases $\phi_{\text {orb }}=0$ (continuous line), and 0.50 (dashed line). (b) All amplitudes $A_{\mathrm{CPS}}$ vs. $\phi_{\mathrm{cyc}}$ (c) Binned amplitudes $A_{\text {cyc,binned }}$ vs. $\phi_{\text {cyc. }}$ (d) Binned standard deviations $S_{\text {cyc,binned }}$ vs. $\phi_{\text {cyc. }}$ (e) All amplitudes $A_{\text {CPS }}$ vs. $\phi_{\text {orb. }}$. (f) Binned amplitudes $A_{\text {orb,binned }}$ vs. $\phi_{\text {orb. }}$ (g) Binned standard deviations $S_{\text {orb,binned }}$ vs. $\phi_{\text {orb. }}$

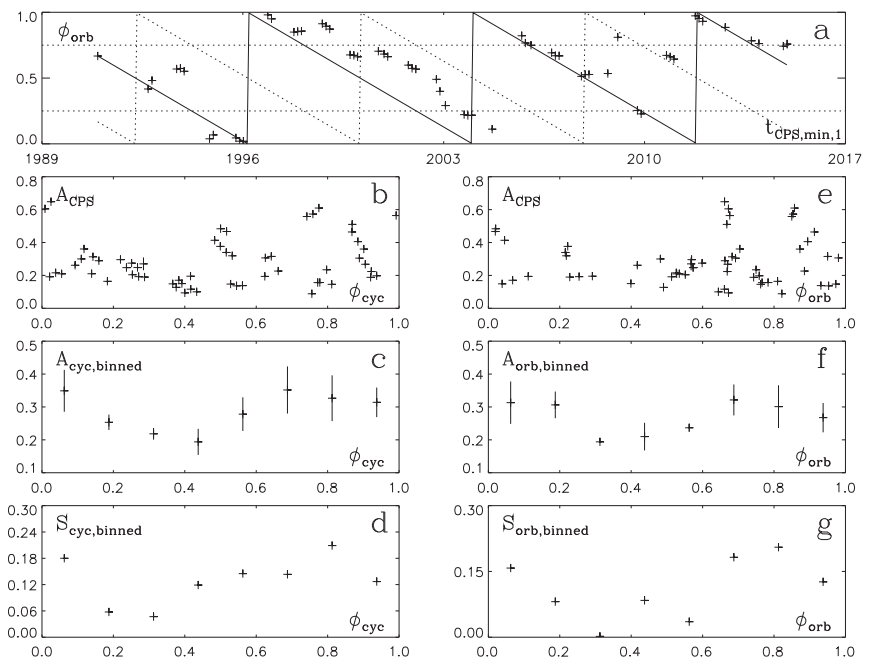

Figure 16. XX Tri; otherwise as in Figure 15.
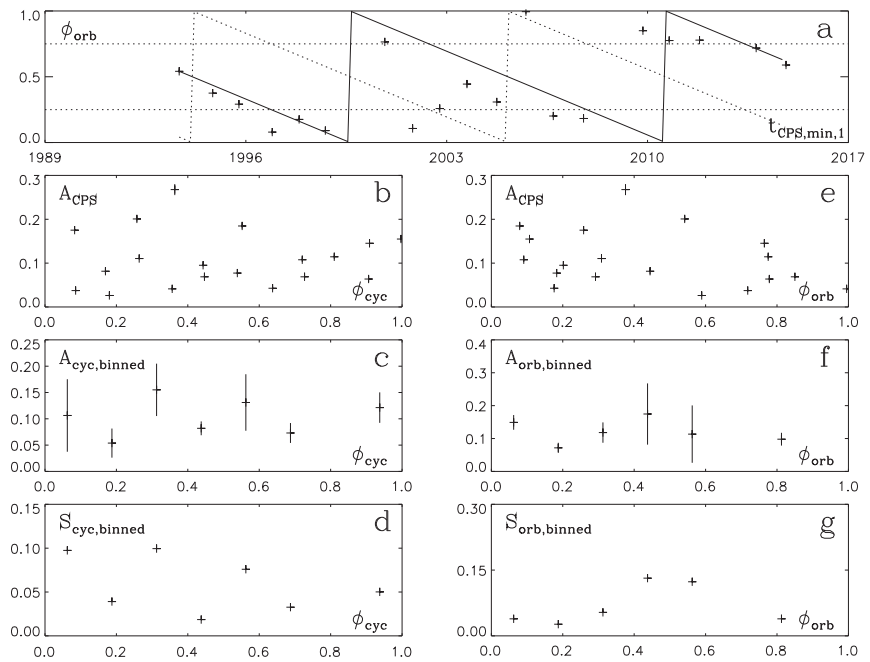

Figure 17. EL Eri; otherwise as in Figure 15. 

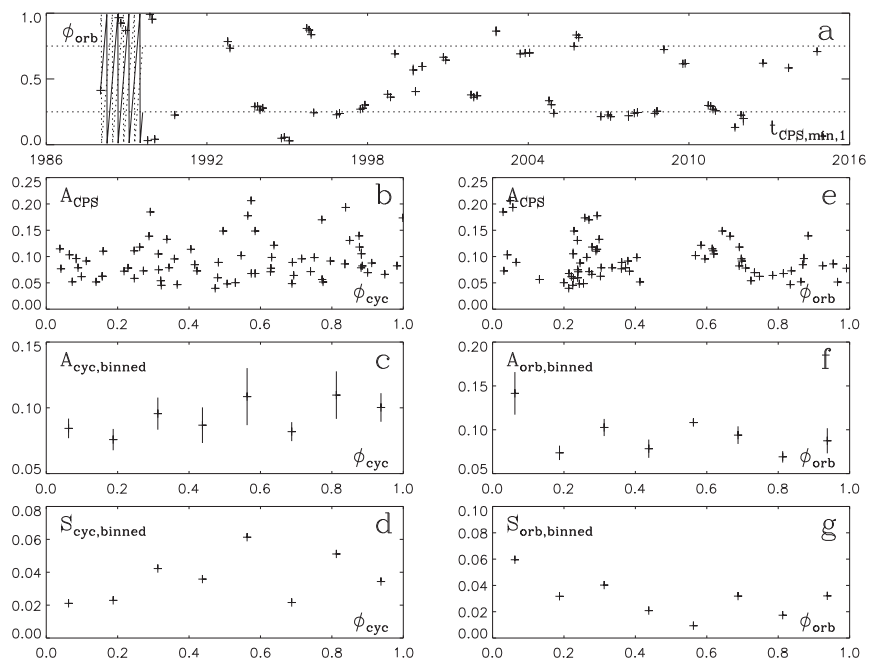

Figure 18. V711 Tau; otherwise as in Figure 15.
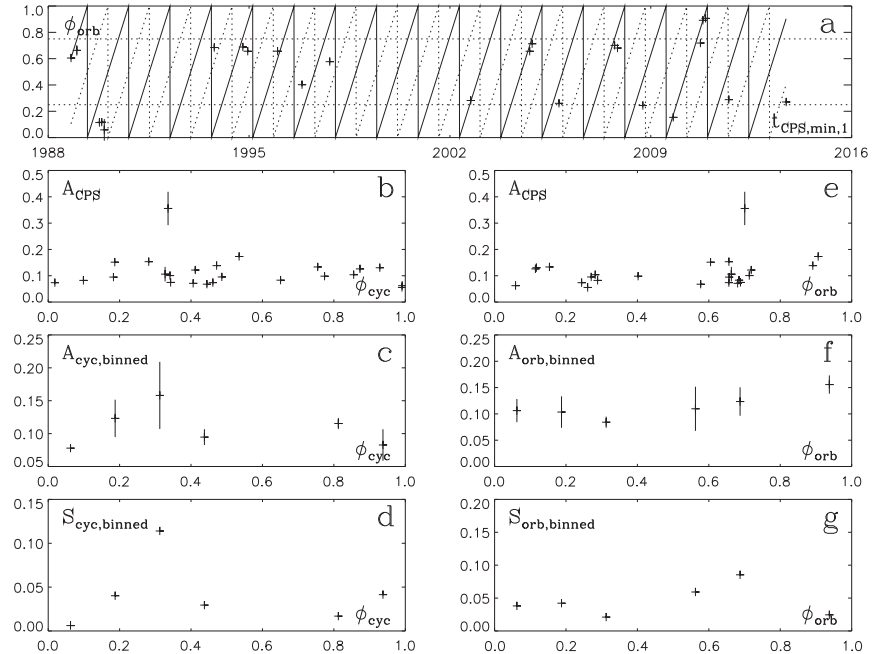

Figure 19. EI Eri; otherwise as in Figure 15.
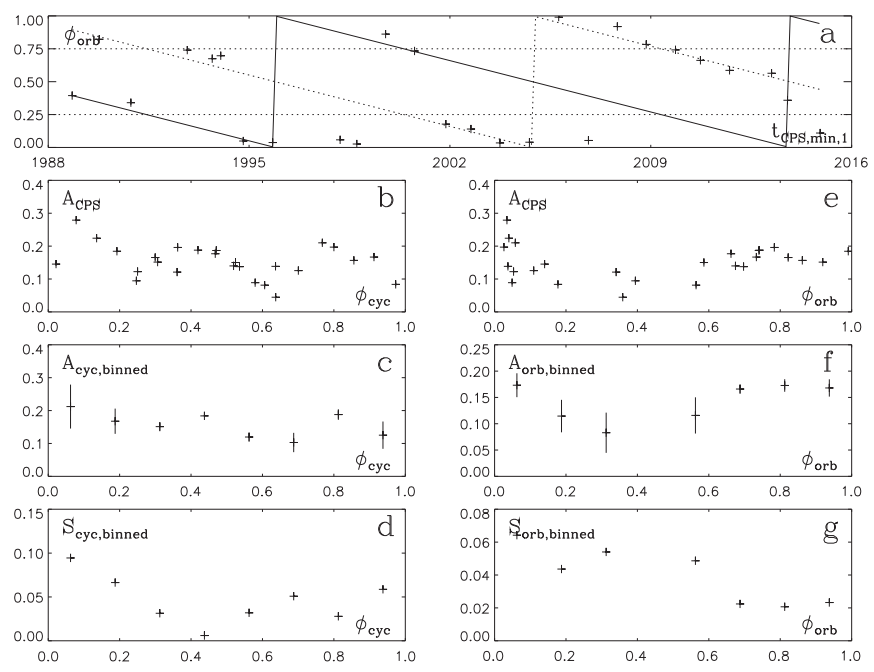

Figure 20. V1149 Ori; otherwise as in Figure 15

secondary minimum $\phi_{\text {orb }}=0.8$ in the first part shifts to 0.7 in the second part. The epochs of Ac and Aa coincide with the MLC primary and secondary minima (Fekel 1983; Donati et al. 1992; García-Alvarez et al. 2003; $P_{\text {orb }}, t_{0}, e$, Sp-type of
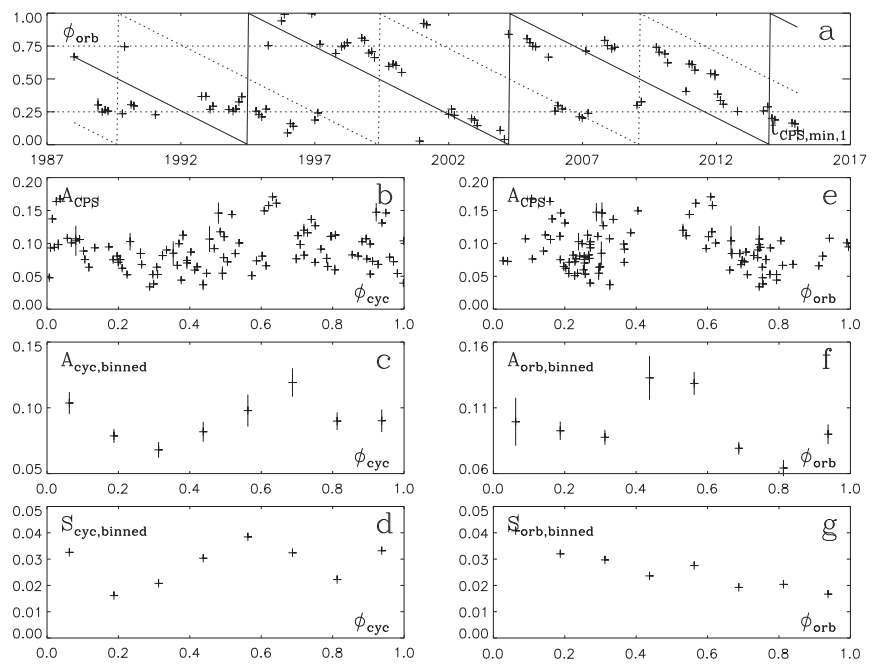

Figure 21. $\sigma$ Gem; otherwise as in Figure 15 .
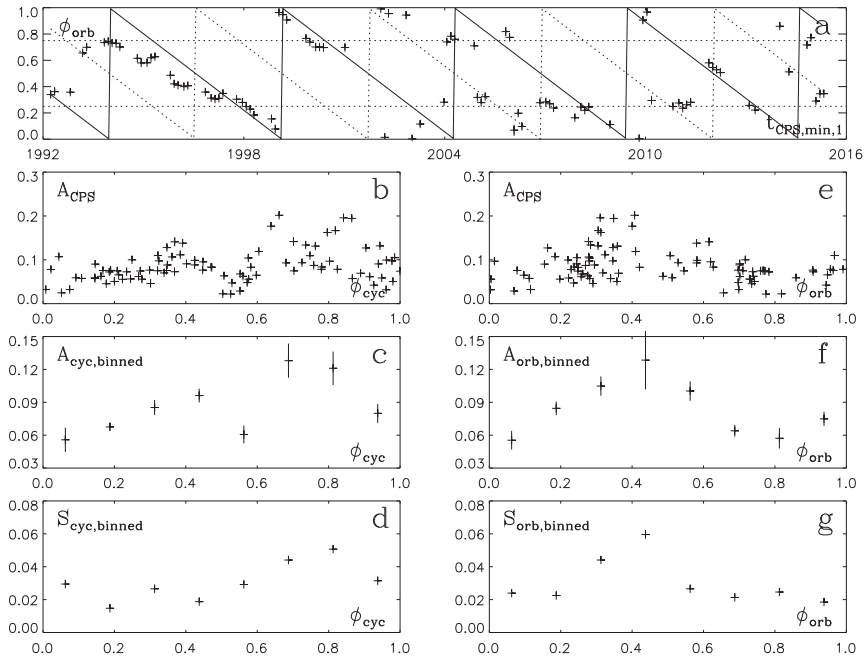

Figure 22. FG UMa; otherwise as in Figure 15.
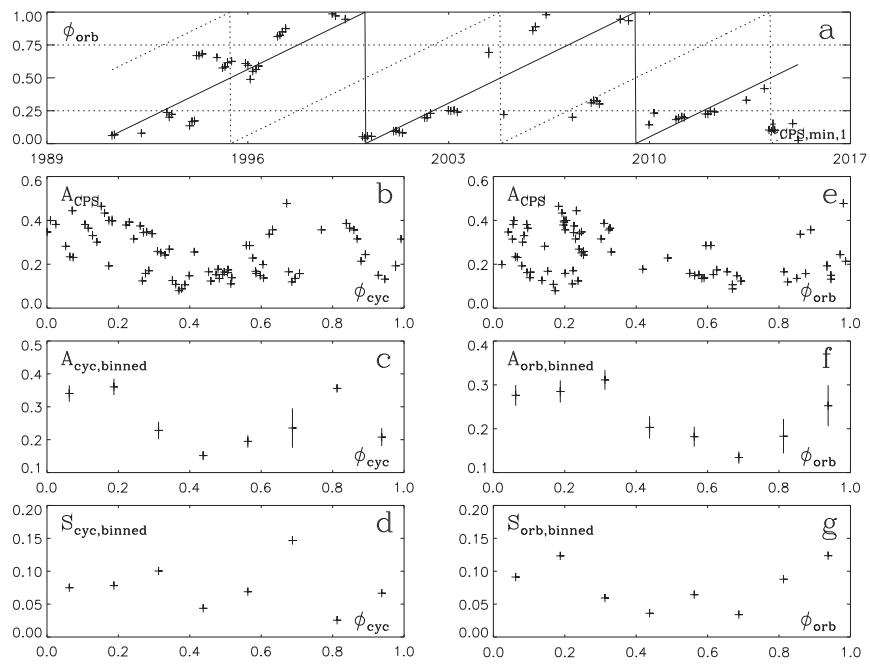

Figure 23. HU Vir; otherwise as in Figure 15.

component A). However, the interpretation of this MLC may be more complicated, because the hotter component B (G5 IV) could also influence MLC (Fekel 1983; García-Alvarez et al. 2003). 

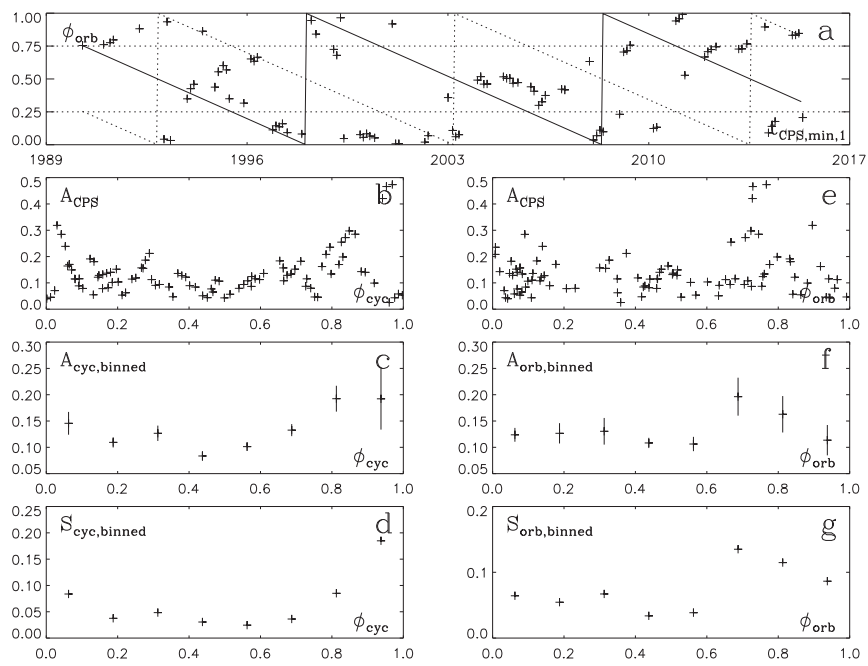

Figure 24. BM CVn; otherwise as in Figure 15.

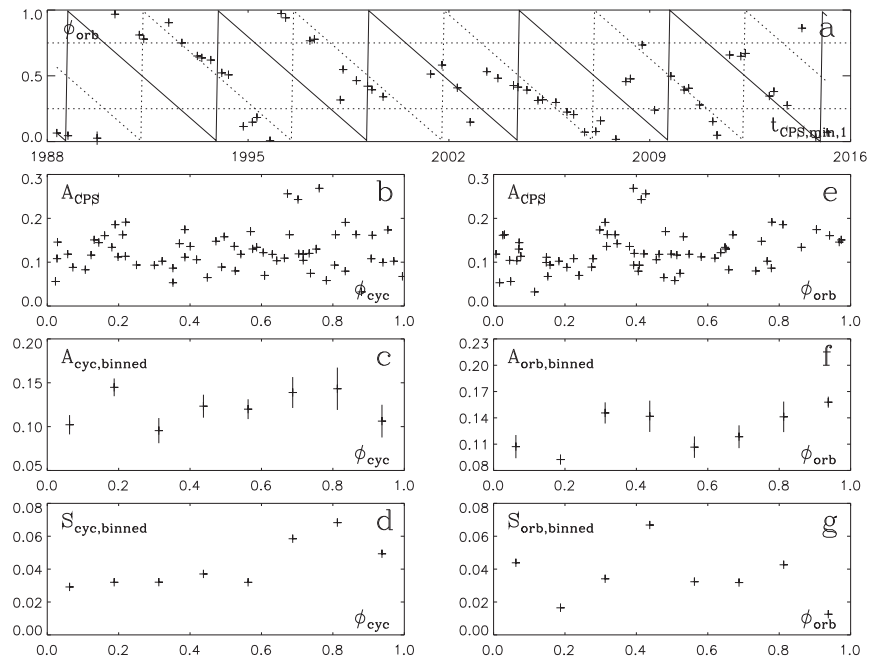

Figure 25. V1762 Cyg; otherwise as in Figure 15.

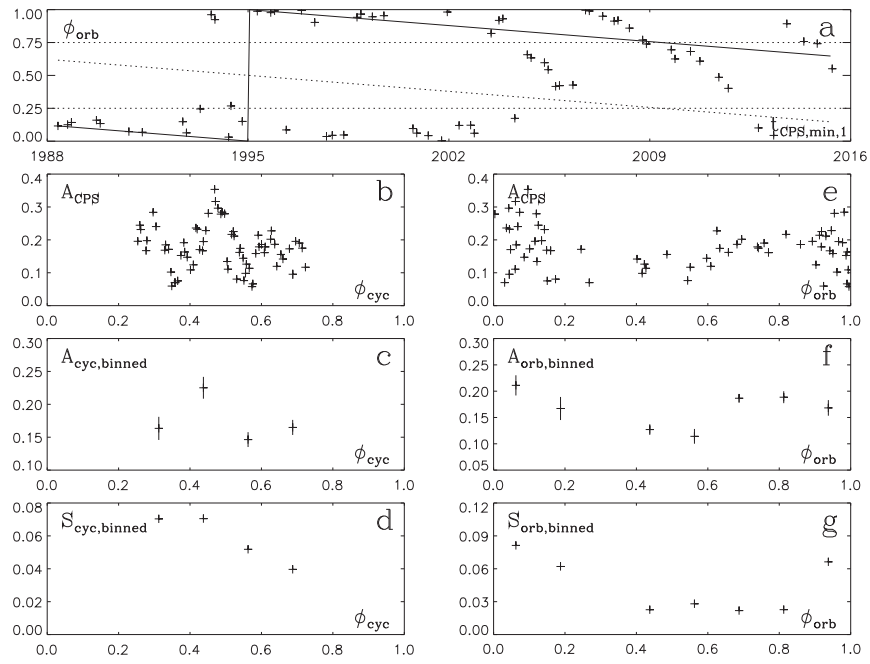

Figure 26. HK Lac; otherwise as in Figure 15.

\subsection{MLC of EI Eri}

EI Eri has a very stable low amplitude double-peaked MLC (Figure 5(a): $A_{\mathrm{All}}=0 \mathrm{~m} \cdot 017$ ). MLC mean and amplitude do not

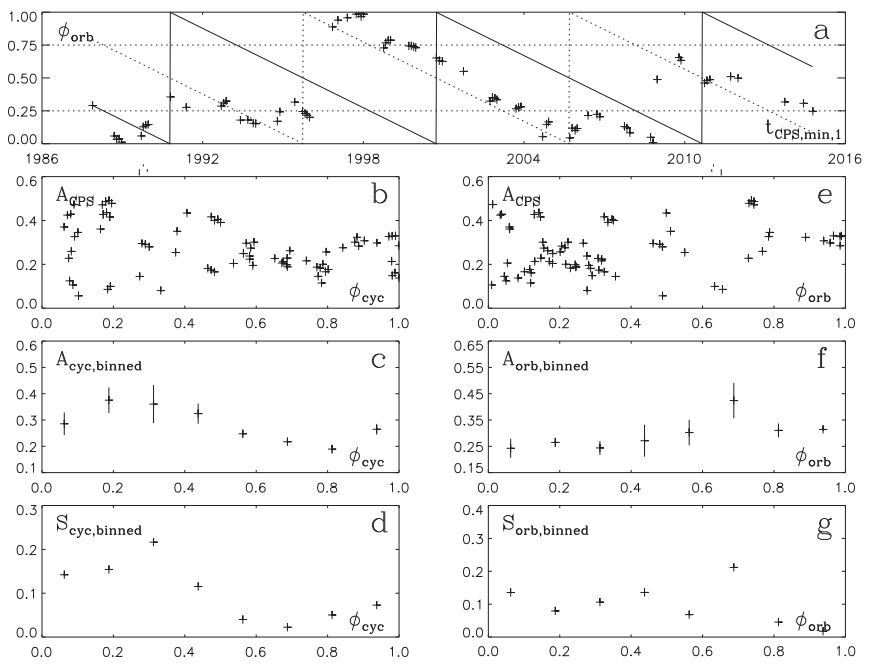

Figure 27. II Peg; otherwise as in Figure 15.

change. Only minor MLC changes occur in the interval $0.25<\phi_{\text {orb }}<0.75$. MLC primary minimum at $\phi_{\text {orb }}=0.65$ in the first part of the data shifts to $\phi_{\text {orb }}=0.20$ in the second part. Epochs of Ac and Aa occur about $\Delta \phi_{\text {orb }}=0.05$ after MLC secondary and primary minima (Fekel et al. 1986, 1987; Strassmeier 1990; Cutispoto 1995; $P_{\text {orb }}, t_{0}, e$, Sp-type of component A).

\subsection{MLC of V1149 Ori}

V1149 Ori has a sinusoidal MLC with an amplitude of $A_{\text {All }}=0.050$ (Figure 6(c)). MLC amplitude, shape, and minimum are stable (Figures 6(c), (e), and (g), $\Delta A=0{ }^{\mathrm{m}}$.012), although MLC mean changes are large (Figures 6(d) and (f)). Epochs of Ac and Aa occur about $\Delta \phi_{\text {orb }}=0.15$ before MLC maximum and minimum (Hall et al. 1991; Fekel \& Henry 2005, $P_{\text {orb }}, t_{0}, e$, Sp-type of component A). Note the MLC dip at about $\phi_{\text {orb }}=0.75$ in Figures 6(c), (e), and (g), which will be discussed later in Section 6.1.4.

\section{7. $M L C$ of $\sigma \mathrm{Gem}$}

$\sigma$ Gem has a stable double-peaked MLC with an amplitude of $A_{\mathrm{All}}=0.039$ (Figure 7, $\Delta A=0.011$ ). MLC mean, amplitude, minimum, and maximum do not change. Small changes are seen only in the interval $0.50<\phi_{\text {orb }}<0.75$. MLC primary and secondary minima coincide with the Ac and Aa epochs (Strassmeier et al. 1988; Bopp \& Dempsey 1989; Duemmler et al. 1997, $P_{\text {orb }}, t_{0}, e$, Sp-type of component A).

\section{8. $M L C$ of FG $U M a$}

FG UMa has a stable second order MLC $(K=2)$. MLC amplitude is about constant $\left(\Delta A=0{ }^{\mathrm{m}} \cdot 010\right)$. The doublepeaked shape and phase of this MLC is the same in Figures 8 (c), (e), and (g), although the mean levels of the first and second part of the $m_{i}$ data are different in Figures 8(d) and (f). The activity level changes only alter the MLC mean, but not the MLC shape, minimum, maximum, or amplitude. MLC primary and secondary minima nearly coincide with Ac and Aa (Fekel et al. 2002, $P_{\text {orb }}, t_{0}, e$, Sp-type of component A). 


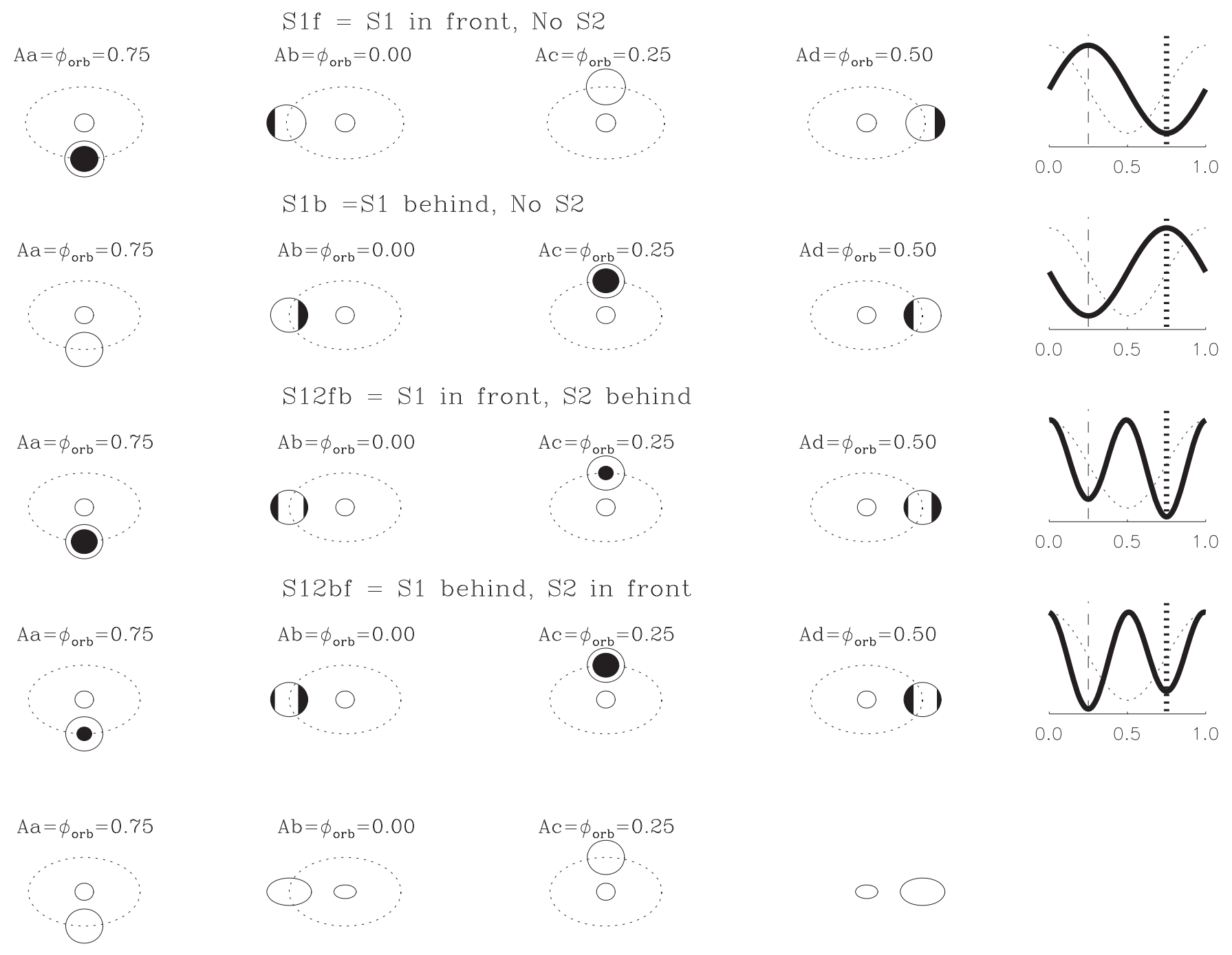













